\title{
Evaluating Usability-Supporting Architecture Patterns: Reactions from Usability Professionals
}

\author{
Edgardo Luzcando, Davide Bolchini, and Anthony Faiola \\ Indiana University Purdue University Indianapolis \\ School of Informatics - HCI \\ \{eluzcand, dbolchin, afaiola\}@iupui.edu
}

\begin{abstract}
Usability professionals and software engineers approach software design differently, which creates a communication gap that hinders effective usability design discussions. An online survey was conducted to evaluate how usability professionals react to Usability-Supporting Architecture Patterns (USAPs) as a potential way to bridge this gap. Members of the Usability Professionals Association (UPA) participated in a pretest-posttest control group design experiment where they answered questions about USAPs and software design. Results suggest that participants perceived USAPs as useful to account for usability in software architectures, recognizing the importance of the USAPs stated usability benefits. Additionally, results showed a difference in perception of the USAPs stated usability benefits between US and European participants. A better understanding of what the usability community thinks about USAPs can lead to their improvement as well as increased adoption by software engineers, which can lead to better integration of usability and HCI principles into software design.
\end{abstract}

Keywords: Architecture Patterns, HCI, Usability, Usability Professionals, Software Design, USAP.

\section{Introduction and Motivation}

The development of modern interactive applications entails the necessity of a smooth coordination and cooperation between software engineers (who conceive, design and develop the technological infrastructure for the system to be) and usability professionals (who conceive, design and develop the elements of the user experience). Due to cultural and historical factors, the tools mastered by software engineers and usability professionals are different and represent their own fields in isolation, thus mining mutual understanding and ultimately posing obstacles to the efficient accomplishment of the goals of the project [1-3].

In particular, one of the common communication breakdowns between software engineers and usability professionals is the lack of strategies to inform the early design of software architectures with usability principles, which helps avoid late (and expensive) architectural changes to accommodate user experience requirements [4]. As a consequence, user requirements are typically added on top of already developed software architectures, thus being constrained and locked early on by system-centered design decisions [5]. Edwards recently warned that we have been successful at 
"covering up ill-suited infrastructure features with interface veneer, but there are limits to how far this can take us." [6] He argues that infrastructure and interaction features need to be jointly designed, and not performed ad-hoc.

To address this challenge, Usability-Supporting Architectural Patterns (USAPs) have been recently proposed as a strategy to systematically embed usability requirements in the early design of software architectures [5]. USAPs are a blend of HumanComputer Interaction (HCI) and Software Engineering (SE) principles that provide a framework to design recurrent software and user requirements (e.g. provide the user a way to undo operations). USAPs are enriched with indications on how these requirements may impact the components of the system architecture, and with examples of how to deal with it at this level. The foreseen benefits of leveraging USAPs in software design are many, including: (a) the opportunity for software engineers to consider and take into account the needs of the user experience in making strategic architectural decision; (b) to provide a shared language between usability professionals and software engineers to discuss design decisions in the light of both system and user requirements; (c) to offer reusable solutions (patterns) which capitalizes on previous design expertise.

Initial studies suggest that USAPs are effective when applied by software engineers [7]. However little is known about USAPs understanding and acceptance by usability professionals $[8,9]$. Acknowledging the proposal of USAPs as an important step towards bridging the communication gap between software engineers and usability professionals, we have conducted a study aimed at assessing the perceived value of USAPs among the community of usability professionals. There is the risk, in fact, that the original value of USAPs (improving mutual understanding) may be weakened amongst usability professionals by the way USAPs are proposed and described: still using concepts, terminology and notation familiar only to software engineers.

The study consisted in a focused online survey administrated to usability professionals and was based on the following multi-part hypothesis:

\section{H.1 - Usability professionals can perceive Usability-Supporting Architecture Patterns} as relevant in their everyday work.

H.2 - Usability professionals consider the usability benefits of Usability-Supporting Architecture Patterns important for their everyday work.

H.3 - If Usability-Supporting Architecture Patterns are communicated in more natural HCI terminology to usability professionals, they can better appreciate the value of Usability-Supporting Architecture Patterns in their everyday work.

The remainder of the paper is organized as follows. Section 2 describes the methods and instrument used to conduct the experiment. Section 3 presents the qualitative and quantitative results. Section 4 covers the discussion of the findings, and section 5 summarizes the paper with concluding statements.

\section{Methods and Instruments}

\subsection{Participants}

This study surveyed a convenience sample of usability professionals from the Indianapolis Usability Professionals Association (UPA) and the Swiss UPA. The sample included approximately 80 participants that have academic training in $\mathrm{HCI}, \mathrm{HCI}$ 
professional experience, or both. The study did not differentiate between professionals and students, but it was expected that most participants would have some degree of professional experience in $\mathrm{HCI}$ or related fields given their involvement with the UPA.

\subsection{Survey Design}

The study is based on a mixed-methods research design to analyze an area where little research has been conducted, following a Concurrent Triangulation Strategy [10] and the quantitative data given higher priority during the analysis. The quantitative portion of the experiment used a Pretest-Posttest Control Group Design [11] with a classic between-subjects design where participants are randomly assigned to any of two groups during the data collection phase.

Participants in the experiment group received a treatment in the form of specific USAP materials consisting of a software design scenario and an USAP example. Participants in the control group did not receive the treatment. A questionnaire format was used for the pretest as well as the posttest, including both quantitative and qualitative questions. Demographic information was solicited after the questionnaire, in addition to the opportunity to provide additional comments.

The survey questions were created leveraging survey design techniques from Dillman [12], and several questions were constructed based on previous questions from Schuman and Presser [13] used to survey attitudes. The online survey was constructed from scratch with a combination of PHP and MySQL technologies available at IUPUI. All data was collected and stored in university infrastructure.

\subsection{Procedure}

The survey introduction provided a brief history about the desire to improve usability in software products. Participants were then given pretest questions to record their existing knowledge and experience. Following the pretest, the treatment introduced USAPs (to the experiment group) and explained how leveraging USAPs could facilitate the communication between usability professionals and software engineers. The treatment provided a software design scenario (canceling a command) describing the communication challenges regarding usability in software design and presented a USAP example. During the posttest, participants were asked to rate the importance of USAP usability benefits from an HCI perspective using a Likert scale. This was done with the nine original USAP usability benefits as well as a newly worded set of usability benefits meant to find if different terminology would improve acceptance. Although all nine USAP usability benefits were rated, the study focused on two

Table 1. USAP Usability Benefit Comparison Design

\begin{tabular}{cll}
\hline & Usability Benefit Original Wording & Usability Benefit New Wording \\
\hline 1 & Accelerates error-free portion & Increases Efficiency \\
2 & Reduces impact of slips & Reduce the impact of errors \\
\hline
\end{tabular}


USAP usability benefits: Accelerates error-free portion, and Reduces the impact of slips, as shown Table 1. An initial pilot study and conversations with HCI peers suggested that these two used terminology that was confusing to a usability professional.

Additional posttest questions explored further perceptions about USAPs and software design, asking participants to state their opinions about USAPs and their potential applications in practice. The survey was designed to flow as one continuous questionnaire where participants were unaware of the difference between the pretest questions or posttest questions.

\section{Results}

From the convenience sample of 80 usability professionals, 67 participants began the survey, 49 completed the pretest and 45 completed the posttest. Of the 45 participants that completed the pretest and posttest only the results of 35 participants were complete and summarized in this section. There were 17 participants in the experiment group and 18 in the control group, and 15 were from the Swiss UPA (Region 1), and 20 from the Indianapolis UPA (Region 2). Of the 34 participants that provided demographic information 20 had a masters, doctorate or post-graduate degree, 12 had a bachelors degree, and 2 did not have any degree. From these, 25 reported six or more years of experience.

When asked to what extent they agreed that usability is an important aspect of software design, all 35 participants agreed, and when asked if they had worked in close contact with software engineers, 28 of 35 participants agreed. When asked to what extent they agreed that USAPs would assist usability professionals identify usability concerns that impact the architecture of a software system, 23 of 35 participants agreed $(66 \%)$.

When asked if they found it challenging to apply usability principles in software design projects, 30 of 35 participants answered yes, and when asked if there is a communication gap between usability professionals and software engineers, 33 of 35 participants answered yes. Additionally, participants volunteered comments about the existence of a communication gap between usability professionals and software engineers, as summarized in Table 2. When participants were asked if they were familiar with any methodologies that would improve communication between usability professionals and software engineers, 21 of 35 answered yes (60\%). In addition, those participants who answered yes where asked to list the known methodologies to substantiate their quantitative answer, and their responses are summarized in Table 3.

Participants were asked to rate the importance of original USAP usability benefits as well as the newly worded version using the following scale: Very Important $=1$, Important=2, Somewhat Important=3, Not Important $=4$, and Don't Know=5. The Don't Know answers were filtered out. The results are summarized in Fig. 1 using the following weighted average: Very Important $=16$, Important=12, Somewhat Important $=8$, and Not Important $=4$. 
Table 2. Identified Reasons for the Communication Gap between Groups ${ }^{1}$

\begin{tabular}{llc}
\hline Answer & Identified Issue & Participants \\
\hline Yes & $\begin{array}{l}\text { Knowledge: software engineers only know software } \\
\text { development and usability professionals only know } \\
\text { usability. They don't know each other's disciplines. }\end{array}$ & \\
& $\begin{array}{l}\text { Core focus in project: software engineers focus on } \\
\text { getting all system parts to work, and usability profes- } \\
\text { sionals only focus on system parts that impact the user }\end{array}$ & 7 \\
& interface. \\
& $\begin{array}{l}\text { Mutual understanding: Both groups struggle to under- } \\
\text { stand each other's needs. }\end{array}$ & 4 \\
& $\begin{array}{l}\text { Awareness: software engineers have not been exposed to } \\
\text { usability and usability professionals have not been ex- }\end{array}$ & 2 \\
& $\begin{array}{l}\text { posed to software engineering. } \\
\text { Process: The software design process is may or may not } \\
\text { include usability. }\end{array}$ & 1 \\
& $\begin{array}{l}\text { Availability of usability people: Not all project benefit } \\
\text { from the participation of usability professionals. }\end{array}$ & 2 \\
& $\begin{array}{l}\text { Stated there is gap, but did not elaborate on the reason. } \\
\text { No gap }\end{array}$ & 2 \\
\hline
\end{tabular}

Table 3. Reported Methods to Improve Communication ${ }^{2}$

\begin{tabular}{lc}
\hline Listed Methods & Participants \\
\hline MILE+ & 2 \\
Open communications (e.g. meetings, workshops) & 10 \\
AWARE & 1 \\
HCI-driven methodologies & 1 \\
Using prototypes and mockups & 3 \\
Software development methodologies & 6 \\
Conceptual Comics & 1 \\
\hline
\end{tabular}

An independent groups $t$ test was used to test the difference in the mean response or rated importance of the target USAP usability benefits Accelerates error-free portion and Reduces impact of slips. Respondents from Region $2(M=1.76)$ showed a lower mean response than those from Region $1(M=2.29), t(30)=2.09, p<.05, r=$ .36 .

The rating of USAP usability benefits also collected qualitative data by asking participants to provide any comments if any of the USAP usability benefits were not clear to them. The targeted USAP usability benefits Accelerates error-free portion and Reduces Impacts of Slips received the most comments, mostly about ambiguous meaning and language that was not familiar. The other (non-targeted) USAP usability benefits did not receive similar comments.

\footnotetext{
${ }^{1}$ Included five additional responses from the pretest that were not part of the 35 clean data sets.

${ }^{2}$ Included three additional responses from the pretest that were not part the 35 clean data sets.
} 


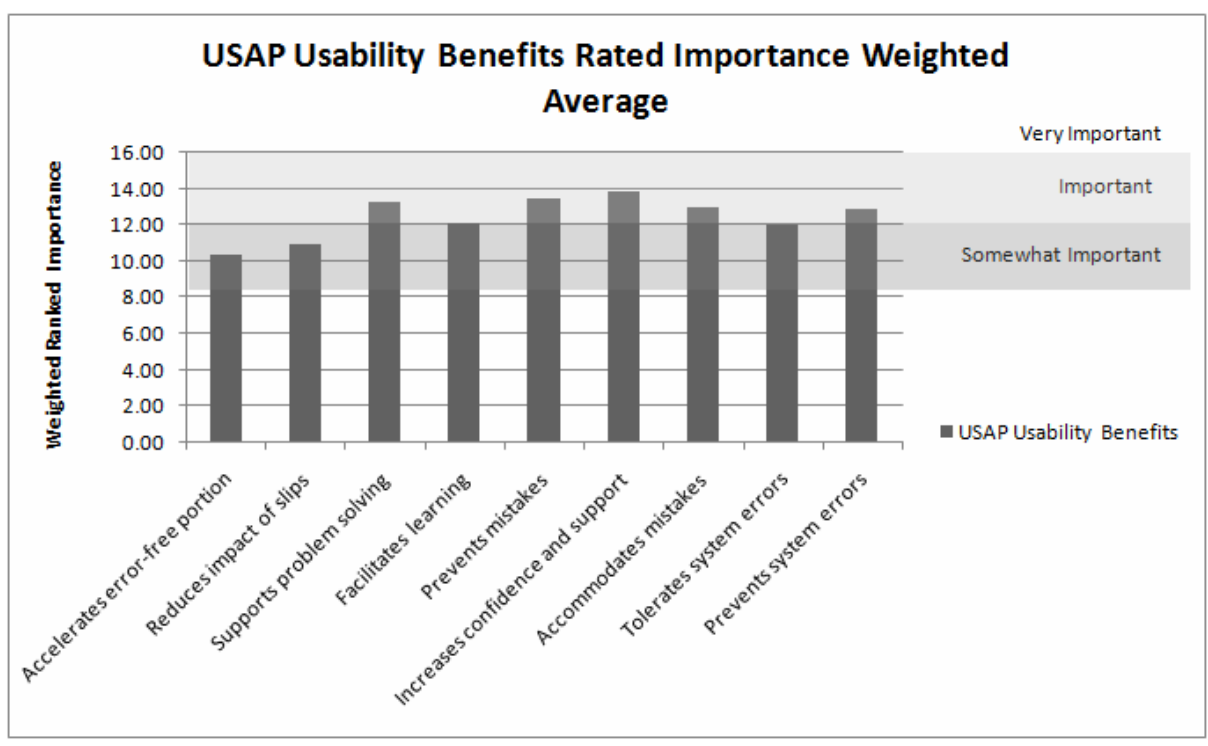

Fig. 1. USAP Usability Benefits Ratings

When asked if they found that leveraging USAPs would be useful for their software design activities, 24 of 35 participants agreed. However, there was a directional difference between the control group and the experiment group. Of the 24 that agreed, 15 were from the control group and 9 were from the experiment group. The experiment group experienced an increase from 0 to 6 participants in the selection of the no opinion choice when compared to the control group.

When asked if there is a communication gap between usability professionals and software engineers, 29 of 35 participants agreed. When participants were asked how likely it would be for them to go and learn more about USAPs after completing the survey, 25 of 35 agreed.

\section{Discussion}

H.1 predicted that usability professionals expect to get benefits of UsabilitySupporting Architecture Patterns in their everyday work. During the pretest $66 \%$ of the participants agreed that USAPs could enable usability professionals identify usability concerns that impact the architecture of a software system. However, it is unclear why $66 \%$ agreed because no participants reported to have a priori knowledge of USAPs, and of the $60 \%$ that reported knowing methodologies to improve this gap, none reported USAPs.

One possible explanation for this result could be that the term "usabilitysupporting" along with "architecture-patterns" could lead to an implicit belief that USAPs are beneficial. In the posttest, $68 \%$ of the participants reported USAPs as 
useful for software design activities based on what they had learned in the survey. However, agreement was directionally different between the control group (62\%) and the experiment group (38\%). This difference could stem from the participants comfort in selecting the no opinion choice.

The selection of the no opinion choice could be an effect of receiving the treatment. It is possible that after participants received the treatment and were exposed to the USAP scenario, they did not understand its purpose or were perhaps confused by the presentation of the materials. For example, it could be that the USAP scenario of canceling a command did not easily apply to their experience, and therefore did not add clarity about the usefulness of USAPs. Conversely, it is possible that participants that did not receive the treatment and did not see the USAP materials were able to imagine (or construct) their own idea of what USAPs are, which in their view might be more effective than the actual USAPs. However, there was no effect found for the treatment in determining the difference between the pretest and posttest difference ( $\mathrm{p}>.10)$.

H. 2 predicted that usability professionals can perceive the importance in using Usability-Supporting Architecture Patterns for their everyday work. During the pretest, $100 \%$ of the participants acknowledged that usability is an important aspect of software design, and $86 \%$ of participants acknowledged they have previously found it challenging to apply usability principles in a software design project. This suggests that participants understood the importance of usability in software design and the challenges of applying usability principles therein. Hence, the fact that $71 \%$ of participants responded that they would likely investigate USAPs further and learn more about them is a potential indication of their usefulness. However, it is possible that the perceived importance of USAPs is a result of recognizing that any technique to improve usability is innately important to usability professionals. This study did not analyze this further.

H.3 predicted that if Usability-Supporting Architecture Patterns are communicated in more natural HCI terminology to usability professionals, they can better appreciate the value of Usability-Supporting Architecture Patterns in their everyday work. We predicted that when participants received the treatment they would rate USAP usability benefits as more important since they had (in the treatment) been exposed to a positive introduction of USAP usability benefits and potential use in software design. The effect of the treatment was non-significant $(p>.10)$ for the ratings.

When contrasting the control group with the experiment group, the targeted USAP usability benefits Accelerates error-free portion and Increases efficiency exhibited an $18 \%$ reduction in rating of importance when compared to their newly worded counterparts Increases Efficiency and Reduce the impact of errors. However, there was no significant effect found for the treatment $(\mathrm{p}=0.63)$.

An unexpected yet interesting result of the experiment was that participants in Region 1 (Europe) responded differently than those in Region 2 (US) when rating the importance of the target USAP usability benefits Accelerates error-free portion and Reduces impact of slips. US usability professionals rated the target USAP usability benefits more important than European usability professionals, which is a potential indication that USAPs are more difficult to understand for European usability professionals than for US usability professionals. 


\section{Conclusion}

This study suggests that usability professionals' initial perception of USAPs is positive. Participants agreed that USAPs are relevant for considering usability concerns in software design, and that usability professionals recognize there is a communication gap with software engineers. However, exposure to USAP materials did not conclusively affect their perception of USAPs. The study suggests that usability professionals generally accept the notion of USAPs without understanding USAP details. This effect was more prominent for US participants in the study, in contrast with their European counterparts. More studies would need to be performed to evaluate additional characteristics of USAPs and their potential acceptance by usability professionals.

\section{Acknowledgments}

Thanks to Dr. Mark Pfaff for his guidance in conducting the statistical analysis for several parts of this study.

\section{References}

1. Snyder, C.: Paper Prototyping: The Fast and Easy Way to Design and Refine User Interfaces. Morgan Kaufmann, San Francisco (2003)

2. Karat, J.: Taking Software Design Seriously. Academic Press, San Diego (1991)

3. Preece, J., Rogers, Y., Sharp, H.: Interaction Design: Beyond Human-Computer Interaction. John Wiley \& Sons, New York (2002)

4. Seffah, A., Gulliksen, J., Desmarais, M.C.: Integrating Usability in the Development Process. In: Seffah, A., Gulliksen, J., Desmarais, M.C. (eds.) Human-Centered Software Engineering: Integrating Usability in the Software Development Lifecycle, pp. 3-14. Springer, Dordrecht (2005)

5. John, B.E., Bass, L., Sanchez-Segura, M.I., Adams, R.: Bringing Usability Concerns to the Design of Software Architecture. In: 9th IFIP Working Conference on Engineering for Human-Computer Interaction and 11th International Workshop on Design, Specification and Verification of Interactive Systems, Hamburg, Germany (2004)

6. Edwards, W.K.: Infrastructure and Its Effect on the Interface. In: Erickson, T., McDonald, D.W. (eds.) HCI Remixed: Reflections on Works That Have Influenced the HCI Community, pp. 119-122. MIT Press, Cambridge (2008)

7. Golden, E., John, B.E., Bass, L.: The value of a usability-supporting architectural pattern in software architecture design: a controlled experiment. In: 27th International Conference on Software Engineering ICSE, St. Louis, Missouri, p. 460 (2005)

8. Adams, R., Bass, L., John, B.E.: Experience with using general usability scenarios on the software architecture of a collaborative system. In: Seffah, A., Gulliksen, J., Desmarais, M.C. (eds.) Human-Centered Software Engineering: Integrating Usability in the Software Development Lifecycle, pp. 87-112. Springer, Dordrecht (2005)

9. John, B.E.: Evidence-Based Practice in Human-Computer Interaction and Evidence Maps. ACM SIGSOFT Software Engineering Notes 30, 1-5 (2005) 
10. Creswell, J.W.: Research Design: Qualitative, Quantitative, and Mixed Methods Approaches. Sage Publications, Thousand Oaks (2003)

11. Campbell, D.T., Stanley, J.C.: Experimental and Quasi-Experimental Designs for Research. Houghton Mifflin Company, Boston (1963)

12. Dillman, D.A.: Mail and Internet Surveys: The Tailored Design Method. John Wiley \& Sons, New York (2000)

13. Schuman, H., Presser, S.: Questions and Answers in Attitude Surveys. Academic Press, New York (1981) 